\title{
Asymmetry and Parity Violation in Magnetoresistance of Magnetic Diluted Dirac-Weyl Semimetal $\left(\mathrm{Cd}_{0.6} \mathrm{Zn}_{0.36} \mathrm{Mn}_{0.04}\right)_{3} \mathrm{As}_{2}$
}

\author{
Oleg Ivanov,* Vasilii Zakhvalinskii, Tatiana Nikulicheva, Maxim Yaprintsev, \\ and Sergei Ivanichikhin
}

Orientation dependences of magnetoresistance, $\rho(\vec{B})$, of single crystal $\left(\mathrm{Cd}_{0.6} \mathrm{Zn}_{0.36} \mathrm{Mn}_{0.04}\right)_{3} \mathrm{As}_{2}$ having tetragonal $\mathrm{P4}_{2} / \mathrm{nmc}$ symmetry have been studied. Three orientations between electrical current, $\vec{\jmath}$, flowing along crystal (100) plane and magnetic field, $\vec{B}$, have been used as follows: 1) $\vec{B}$ is perpendicular to both $\vec{J}$ and (100) plane; 2) $\vec{B}$ is perpendicular to $\vec{J}$ but parallel to (100) plane; 3) $\vec{B}$ is parallel to both $\vec{J}$ and (100) plane. Asymmetry in curves $\rho(\vec{B})$ is observed for all orientations. For orientation (2), crossover from negative to positive magnetoresistance is found as magnetic field changes its direction from one position corresponding to negative $\vec{B}$ to opposite direction corresponding to positive $\vec{B}$. So magnetoresistance parity typical for numerous solids is violated for this orientation. The Shubnikov-de Haas $(\mathrm{SdH})$ oscillations are observed for all orientations above $\approx 1.8 \mathrm{~T}$ and below $\approx 40 \mathrm{~K}$. The effective mass of electrons extracted from $\mathrm{SdH}$ oscillations analysis is found to be orientation dependent. Smeared kinks correlated with the $\mathrm{SdH}$ oscillations are also observed in the Hall effect study for orientation (1). Magnetoresistance peculiarities can be related to specific properties of the Dirac semimetals and their evolution under magnetic field. demonstrating unusual features in transport properties like giant positive magnetoresistance, non-trivial quantum oscillations of the electrical resistivity and Landau level splitting in magnetic field. ${ }^{[6-12]}$ The 3D topological Dirac semimetals are important class of topological insulators. ${ }^{[13]}$ The Dirac point (or Dirac node) in the 3D Dirac semimetal is originated from overlapping two Weyl nodes with opposite chirality in momentum space. The breaking of either time reversal symmetry or inversion symmetry in the Dirac semimetal can remove the Dirac nodes degeneracy resulting in the Weyl semimetal phase with opposite chiral Weyl node pairs. Moreover, novel transport phenomena can be originated from the non-conserving chiral current between separated Weyl nodes. ${ }^{[14-18]}$ Time reversal symmetry in $\mathrm{Cd}_{3} \mathrm{As}_{2}$ can be naturally broken under external magnetic field, that results in a transition from a Dirac semimetal to a Weyl semimetal. ${ }^{[19]}$

Magnetoresistance, $\rho(\vec{B})$, characterizing a change of the electrical resistivity, $\rho$, of solid in applied magnetic field, $\vec{B}$, is a galvanomagnetic effect possessing a parity regarding a sign change of magnetic field. ${ }^{[1]}$ That is, firstly, $\rho(\vec{B})$ does not change its sign, if the $\vec{B}$ reorientation of from some initial direction to opposite one occurs. Secondly, the $\rho(\vec{B})$ curves are symmetric regarding to the $\rho$ axis. ${ }^{[2-5]}$ It means that the magnetoresistance is an even field function satisfactory to the $\rho(+B)=\rho(-B)$ condition. In turn, the magnetoresistance sign can be positive or negative dependent on magnetoresistance mechanism.

$\mathrm{Cd}_{3} \mathrm{As}_{2}$ is believed to be as one of solids, in which the magnetoresistance parity could be violated under specific conditions. This compound is a 3D topological Dirac semimetal with inverted bands and ultra-high electron mobility,

Prof. O. Ivanov, Prof. V. Zakhvalinskii, Dr. T. Nikulicheva,

Dr. M. Yaprintsev, S. Ivanichikhin

Belgorod State University

Pobedy St. 85, Belgorod 308015, Russia

E-mail: ivanov.oleg@bsu.edu.ru

The ORCID identification number(s) for the author(s) of this article can be found under https://doi.org/10.1002/pssr.201800386.

DOI: 10.1002/pssr.201800386 semimetals are intensively studied. The $\rho(\vec{B})$ curves symmetric regarding to the $\rho$ axis usual for a lot of solids were found in $\mathrm{Cd}_{3} \mathrm{As}_{2}, \mathrm{Nb}(\mathrm{Ta}) \mathrm{P}(\mathrm{As}), \mathrm{Nb}(\mathrm{Ta}) \mathrm{P}(\mathrm{As}, \mathrm{Sb})_{2}, \mathrm{ZrSiS}, \mathrm{Bi}_{1-x} \mathrm{Sb}_{x}, \mathrm{Bi}$, $\mathrm{Na}_{3} \mathrm{Bi}$, GdPtBi. ${ }^{[20-24]}$ However, in needle $\mathrm{Cd}_{3} \mathrm{As}_{2}$ crystal with its long axis along [112] crystal direction, the antisymmetric $\rho(\vec{B})$ curves were also found. ${ }^{[25]}$ This antisymmetry indicating to the violation of the magnetoresistance parity could be likely taken as one of fundamental properties of topological insulators. So, actual scientific task is to search other topological insulators demonstrating the violation of the magnetoresistance parity and find specific material properties resulting in this violation.

The aim of this paper is to introduce the $\left(\mathrm{Cd}_{0.6} \mathrm{Zn}_{0.36} \mathrm{Mn}_{0.04}\right)_{3} \mathrm{As}_{2}$ single crystal as the $3 \mathrm{D}$ topological semimetal demonstrating unusual magnetotransport properties including the violation of the magnetoresistance parity. As will be discussed below, this compound has a few specific features, compounds and, secondly, seem to be important to result in unusual properties presented in this paper.

To grow the $\left(\mathrm{Cd}_{0.6} \mathrm{Zn}_{0.36} \mathrm{Mn}_{0.04}\right)_{3} \mathrm{As}_{2}$ single crystal, the modified Bridgeman method was applied. A melt consisting
Currently, the magnetic properties of various Dirac and Weyl which, firstly, make it different from many other $\mathrm{Cd}_{3} \mathrm{As}_{2}$ 
of stoichiometric $\mathrm{Cd}_{3} \mathrm{As}_{2}, \mathrm{Zn}_{3} \mathrm{As}_{2}$, and $\mathrm{Mn}_{3} \mathrm{As}_{2}$ amounts was slowly cooled at $5{ }^{\circ} \mathrm{Ch}^{-1}$ rate within the temperature range from the melting temperature at $1113 \mathrm{~K}$ down to the $\alpha-\beta$ phase transition temperature at $738 \mathrm{~K}$ under the temperature $2 \mathrm{~K} \mathrm{~cm}^{-1}$ gradient.

Elemental composition of the single crystal corresponding to the $\left(\mathrm{Cd}_{0.6} \mathrm{Zn}_{0.36} \mathrm{Mn}_{0.04}\right)_{3} \mathrm{As}_{2}$ stoichiometry was derived by a Shimadzu ICP (inductively coupled plasma) emission spectrometer ICPE-9000.

XRD analysis carried out by a Rigaku Ultima IV diffractometer verified the high crystallinity of the single crystal. To determine a space symmetry group of the single crystal studied, XRD analysis of powder prepared by grinding this single crystal was also carried out. XRD pattern of the powder prepared by grinding the single crystal is shown in Figure S1, Supporting Information. All the diffraction peaks can be exactly indexed with the standard diffraction planes corresponding to tetragonal $\mathrm{P} 4_{2} /$ nmc symmetry (ICDO Card No. 03-065-857) with unit cell parameters equal to $a=0.8752 \mathrm{~nm}$ and $c=1.2344 \mathrm{~nm}$.

The plate-like rectangular crystal with dimensions of $0.5 \times$ $1.0 \times 5.0 \mathrm{~mm}^{3}$ was prepared to measure $\rho$ and examine the Hall effect by a Mini Cryogen Free Measurements System (Cryogenic Ltd., UK). The $\rho$ measurements were carried out by reversing the measuring current.

A larger surface of the crystal being studied was oriented parallel to crystal (100) plane as confirmed by XRD pattern shown in the inset of Figure 1.

Magnetic field dependences of $\rho$ were measured at three various orientations between electrical current, $\vec{J}$, flowing along crystal (100) plane and magnetic field. These orientations shown by scheme in the top of Figure 1 are corresponding to: 1) $\vec{B}$ is perpendicular to both $\vec{J}$ and (100) plane; 2 ) $\vec{B}$ is perpendicular to $\vec{J}$ but parallel to (100) plane; 3 ) $\vec{B}$ is parallel to both $\vec{J}$ and (100) plane.

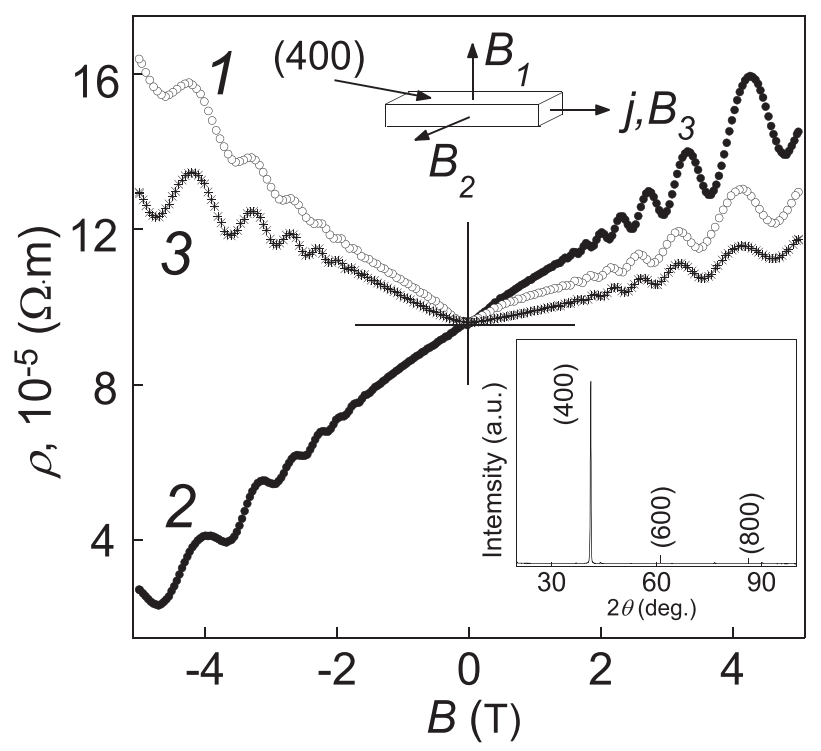

Figure 1. The $\rho$ versus $\vec{B}$ dependences taken at $2 \mathrm{~K}$ for various orientations corresponding to scheme in the top. XRD pattern of plate-like rectangular crystal used in this experiment is shown in the inset.
The $\rho(\vec{B})$ curves taken for all the orientations at temperature of $2 \mathrm{~K}$ are also shown in Figure 1. A few peculiarities can be easily found in these curves as follows: a) the magnetoresistance is anisotropic since both magnitude and shape of the $\rho(\vec{B})$ curves are orientation dependent; b) the Shubnikov-de Haas ( $\mathrm{SdH}$ ) oscillations due to the formation of Landau levels are observed for all the orientations at high enough magnetic fields above $\approx 1.8 \mathrm{~T}$; c) the $\rho(\vec{B})$ curves are asymmetric, since these curves taken at the $\vec{B}$ sweeping in one direction corresponding to the positive fields are remarkably different from the $\rho(\vec{B})$ curves recorded at the $\vec{B}$ sweeping in opposite direction corresponding to the negative fields; d) the $\rho(\vec{B})$ dependences taken for the 1 and 3 orientations although are asymmetric, however, there are positive for both positive and negative $\vec{B}$; e) in contrast to the 1 and 3 orientations, the $\rho(\vec{B})$ dependence taken for the 2 orientation is positive at the $\vec{B}$ sweeping in the positive direction, but it is negative at the $\vec{B}$ sweeping in the negative direction. In other words, for this measurement orientation a crossover from positive to negative magnetoresistance occurs as magnetic field is changed from initial positive direction to opposite negative one. So, magnetoresistance becomes an odd field function, $\rho(+B)=-\rho(-B)$.

The $\mathrm{SdH}$ oscillations shown in Figure 1 are observed up to $\approx 40 \mathrm{~K}$, while the $\mathrm{SdH}$ amplitudes are gradually decreasing with increasing temperature. According to the Lifshitz-Kosevich theory, the temperature dependence of the SdH amplitudes, $A$, can be expressed as ${ }^{[26]}$

$$
A \sim \frac{X}{\sinh (X)} \exp \left[\frac{-2 \pi^{2} m^{*} k_{\mathrm{B}} T_{\mathrm{D}}}{\hbar e B}\right]
$$

with $X=\left(2 \pi^{2} k_{\mathrm{B}} T m^{*} /(\hbar e B)\right.$, and where $T_{\mathrm{D}}$ is the Dingle temperature, $m^{*}$ is the effective cyclotron mass of the electron, $\hbar$ is the Planck constant, $k_{\mathrm{B}}$ is the Boltzmann constant, $e$ is the electron charge, and $m_{0}$ is the free electron mass.

Next, the $A$ values measured at two different temperatures (2 and $4 \mathrm{~K}$ ) but at the same magnetic field were used to estimate $m^{*}$ by expression (1). The $m^{*}$ estimates for different measurement orientations calculated for zero magnetic field are listed in Table 1 . To account for various direction of $\vec{B}$, additional letter symbols like (a)-(f) were also introduced. It was found that for the (a), (b), (d), and (e) orientations, $m^{*}$ is weakly orientation dependent and equal to $\approx 0.04 m_{0}$. This estimate is in well accordance with the $m^{*}$ values reported earlier. ${ }^{[2]}$ However, for the (c) and (f) orientations $m^{*}$ is extremely less $\left(m^{*} \approx 0.018 m_{0}\right)$.

Table 1. Parameters estimated from the $\mathrm{SdH}$ oscillations for various $\vec{B}$ and $\vec{J}$ orientations.

\begin{tabular}{lllcrcc}
\hline Orientation & & & $m^{*} / m_{0}$ & $T_{\mathrm{D}}(\mathrm{K})$ & $\tau \times 10^{13}(\mathrm{~s})$ & $\alpha / m_{0} \times 10^{3}\left(\mathrm{~T}^{-1}\right)$ \\
\hline $1: \vec{B}_{1} \perp \vec{J}$ & $B_{+}$ & (a) & 0.042 & 12.40 & 0.99 & 3.5 \\
& $B_{-}$ & (b) & 0.043 & 11.80 & 1.04 & 9.0 \\
$2: \vec{B}_{2} \perp \vec{J}$ & $B_{+}$ & (c) & 0.018 & 9.74 & 1.26 & 53.6 \\
& $B_{-}$ & (d) & 0.040 & 9.31 & 1.32 & 0 \\
$3: \vec{B}_{3} \| \vec{J}$ & $B_{+}$ & (e) & 0.041 & 11.00 & 1.12 & 0 \\
& $B_{-}$ & (f) & 0.017 & 8.50 & 1.45 & 6.8 \\
\hline
\end{tabular}


Moreover, $m^{*}$ was found to be $\vec{B}$ dependent for the (a)-(c) and (f) orientations, and in this case the $m^{*}(B)$ dependences obey a linear $m^{*}(B)=m^{*}(0)+a B$ law. The $a / m_{0}$ coefficients for various orientations are also listed in Table 1.

The Dingle temperature was found from the slope in the semilog plot of $\left[A B^{1 / 2} \sinh (X) / X\right]$ versus $1 / B$ taken at $2 \mathrm{~K}$ (see Table 1). The $T_{\mathrm{D}}$ temperature is related to the quantum lifetime of carriers, $\tau$, as $T_{\mathrm{D}}=\hbar / 2 \pi k_{\mathrm{B}} \tau$. Since $T_{\mathrm{D}}$ was found to be $\vec{B}$ dependent, $\tau$ due to the carriers scattering is $\vec{B}$ dependent, too.

Finally, $\mathrm{SdH}$ electron concentration, $n_{\mathrm{SdH}}$, was extracted by expression

$n_{\mathrm{SdH}}=1 /\left[3 \pi^{2}(2 e / \hbar)^{3 / 2}\left(1 / P_{\mathrm{SdH}}\right)^{3 / 2}\right]$

where $P_{\mathrm{SdH}}$ is the period of the $\mathrm{SdH}$ oscillations.

The $n_{\mathrm{SdH}}$ value estimated as $3.24 \cdot 10^{17} \mathrm{~cm}^{-3}$ was naturally independent on the measurement orientation.

Details of the $\mathrm{SdH}$ oscillations analysis are presented in Figure S2, Supporting Information.

It is known that the quantum Hall effect correlated with the SdH effect should be observed in 3D topological insulators. ${ }^{[28,29]}$ The quantum Hall effect is characteristic of 2D electron system. ${ }^{[30]}$ Two types of the carriers corresponding to $2 \mathrm{D}$ and 3D electrons are coexisting in topological insulators. The matter is that owing to the interplay between the topology of the electronic band structure and strong spin-orbit coupling in the bulk, the surface state of topological insulators give rise to $2 \mathrm{D}$ Dirac fermions. In contrast to the gapped bulk states, the edge or surface states are gapless. Under external magnetic field, the continuous density of states of the 2D electron gas will split into equally spaced Landau levels. When the Fermi level of the system lies between two neighboring Landau levels, the bulk carriers are localized. However, the electrons can propagate along the edge of the sample. In this case, the Hall voltage will form well-defined plateaus and the longitudinal resistance becomes exactly zero.

The smeared plateaus observed as kinks in the Hall voltage, $U_{\mathrm{H}}$, were actually found in $\left(\mathrm{Cd}_{0.6} \mathrm{Zn}_{0.36} \mathrm{Mn}_{0.04}\right)_{3} \mathrm{As}_{2}$ for the 1 orientation as shown by arrows in the inset of Figure 2. It should be noted that the Hall voltage sign is negative, since the electrons are majority carriers in the compound being studied.

To distinguish these kinks in detail, the $d U_{\mathrm{H}} / d B$ derivative versus $B$ dependence was plotted (curve 1 ) and compared to the $\rho(B)$ dependence. Both dependences taken at the same temperature of $2 \mathrm{~K}$ are corresponding to positive magnetic field. Like the $\mathrm{SdH}$ oscillations, a set of the oscillations is also observed in the $d U_{\mathrm{H}} / d B$ versus $B$ curve, and the amplitude of these oscillations is gradually increasing with increasing magnetic field. There is a clear correlation between the Hall voltage and $\mathrm{SdH}$ oscillations. By comparing the curves 1 and 2 in Figure 2, one can conclude that each extreme (minimum or maximum) in the $\mathrm{SdH}$ oscillations is shifted regarding to the extreme positions in the $d U_{\mathrm{H}} / d B$ oscillations. That is, each peak of the $\mathrm{SdH}$ oscillations is located approximately in the middle between neighboring extremes of the $d U_{\mathrm{H}} / d B$ oscillations. To show this shifting, dashed lines for the extremes in the $\mathrm{SdH}$ oscillations and dotted lines for the extremes in the $d U_{\mathrm{H}} / d B$ oscillations were additionally plotted in Figure 2. It should be noted that for the ideal 2D electron system, the quantum Hall effect is reflected in

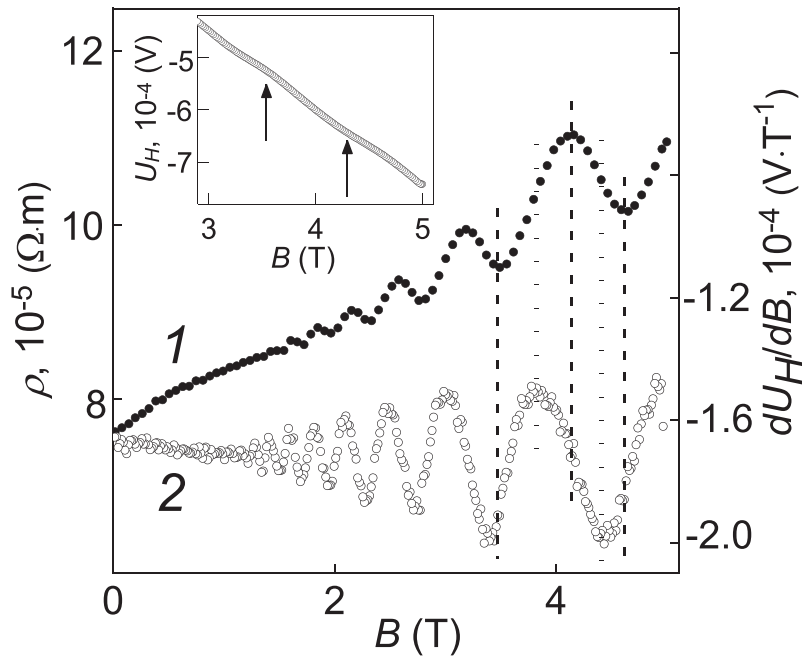

Figure 2. The $d U_{\mathrm{H}} / d B$ versus $B$ (curve 1) and $\rho$ versus $B$ (curve 2) dependences taken at $2 \mathrm{~K}$ for the 1 orientation at positive magnetic field. The $U_{H}$ versus $B$ dependence taken above $3 \mathrm{~T}$ is shown in the inset.

forming the ideal $U_{\mathrm{H}}$ plateaus located parallel to the abscissa axis. However, as was mentioned above, there are two types of the carriers in 3D topological insulators. One of them involved in 2D carrier pairing shows plateaus in the quantum Hall effect and the other one shows 3D behavior. The Hall effect study should reflect their properties in parallel resulting in smearing the $U_{\mathrm{H}}$ plateaus due to the 2D carriers group.

The most intriguing finding reported above is a crossover from the negative to positive magnetoresistance for the 2 orientation as magnetic field changes its direction from one position corresponding to negative $\vec{B}$ to opposite direction corresponding to positive $\vec{B}$ (Figure 1). It is important to note that such kind of the $\vec{B}$ effect on $\rho$ was observed up to $150 \mathrm{~K}$ (Figure 3). This temperature was maximum in our experiments. One can see that within the temperature $2-150 \mathrm{~K}$ interval, $\rho$ is increasing for the positive magnetic field, but it is decreasing for the negative magnetic field as compared to the $\rho$ values taken for zero magnetic field. The temperature dependences of MR calculated as $\mathrm{MR}=[\rho(B)-\rho(0)] / \rho(0)$ for $B=+5 \mathrm{~T}$ (curve 1 ) and $B=-5 \mathrm{~T}$ (curve 2) are presented in the inset of Figure 3 . These dependences are rather complicated. Particularly, maximum in curve 1 and minimum in curve 2 are observed below $50 \mathrm{~K}$.

To explain the antisymmetric magnetoresistance observed earlier in needle $\mathrm{Cd}_{3} \mathrm{As}_{2}$ crystal, two mechanisms were proposed. ${ }^{[25]}$ First of them results from the Hall resistance picked up from macroscopically distorted current paths due to local variations in stoichiometry of the inhomogeneous semiconductors. ${ }^{[30-32]}$ In this case, a perpendicular current component flowing normal to the major current direction can be originated. In turn, this component will result in linear-in- $B$ Hall resistance contribution to the total magnetoresistance. However, reversing the measuring current was applied to take the $\rho(\vec{B})$ (Figure 1) and $\rho(T)$ (Figure 3) dependences. So, the Hall resistance contribution to the total magnetoresistance was minimized. Besides, the SdH oscillations were clearly observed above $\approx 1.8 \mathrm{~T}$ and up to $\approx 40 \mathrm{~K}$ in $\left.\mathrm{Cd}_{0.6} \mathrm{Zn}_{0.36} \mathrm{Mn}_{0.04}\right)_{3} \mathrm{As}_{2}$. These oscillations can be as a rule observed in perfect single crystals 


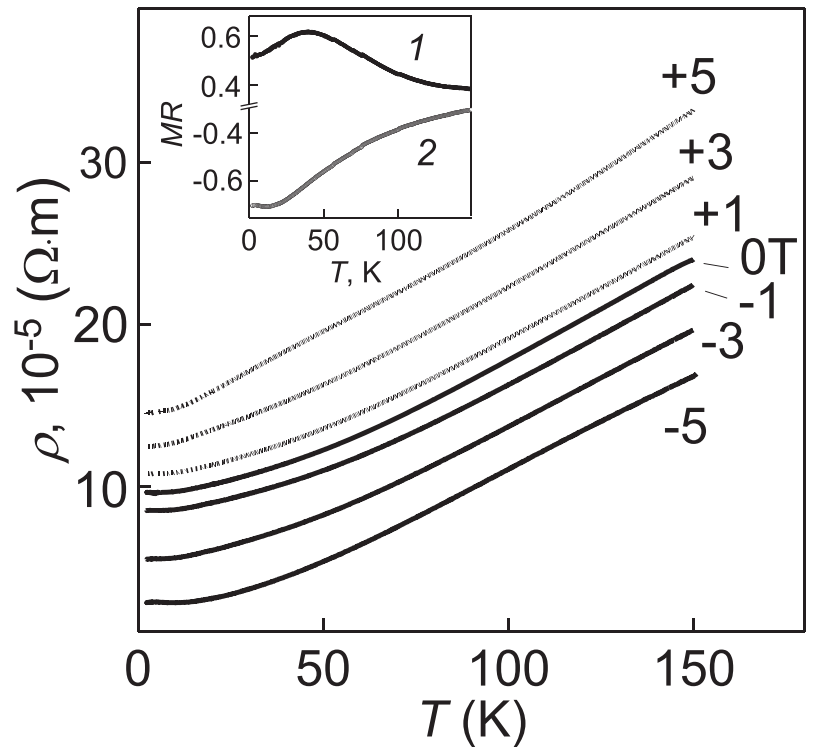

Figure 3. The $\rho$ versus $\vec{B}$ dependences for the 2 orientation taken at various magnetic field values indicated in the right of each curve. The MR versus $B=+5 \mathrm{~T}$ (curve 1 ) and $\mathrm{MR}$ versus $B=-5 \mathrm{~T}$ (curve 2 ) dependences are shown in the inset.

having a minimum amount of various defects. The inhomogeneous semiconductors should have a lot of various defects preventing the $\mathrm{SdH}$ oscillations appearance.

Another mechanism explaining the antisymmetric magnetoresistance in needle $\mathrm{Cd}_{3} \mathrm{As}_{2}$ crystal takes into account the anomalous velocity term associated with the non-zero Berry curvature of the bulk band. ${ }^{[25]}$ Generally, the electron velocity, $\vec{v}$, can be expressed as ${ }^{[23]}$

$\hbar \vec{v}=\nabla_{\mathrm{k}} \epsilon(k)+e \vec{E} \times \vec{\Omega}(\vec{B}, \vec{k})$

where $\epsilon$ is the electron energy, $\vec{E}$ is the electric field, $\vec{k}$ is the wave vector of the electron, and $\vec{\Omega}(\vec{B}, \vec{k})$ is the Berry curvature.

Then, a total current at a diffusive transport is given by

$\vec{J}=\int \frac{d^{3} \vec{k}}{(2 \pi)^{3}}\left|\vec{v}+e \vec{E} \times \vec{\Omega}(\vec{B}, \vec{k})+\frac{e}{c}(\vec{\Omega}(\vec{B}, \vec{k}) \cdot \vec{v}) \vec{B}\right| n_{\vec{k}}$

where $n_{\vec{k}}=f_{\vec{k}}^{0}+g_{\vec{k}}(\vec{B}, \vec{E})$ is the electron distribution function, and $f_{\vec{k}}^{0}$ and $g_{\vec{k}}(\vec{B}, \vec{E})$ are the equilibrium and non-equilibrium distribution function, respectively.

In zero magnetic field, time reversal symmetry will be valid, that corresponds to a Dirac semimetal. In this case, the integration of anomalous velocity term $(e \vec{E} \times \vec{\Omega}(\vec{B}, \vec{k}))$ with $f_{\vec{k}}^{0}$ over whole Fermi surface will give zero contribution to the total current. But as was mentioned above, time reversal symmetry can be broken under external magnetic field, which results in a Weyl semimetal phase. Now, the induced $g_{\vec{k}}(\vec{B}, \vec{E})$ term will result in non-vanishing anomalous velocity contribution to the total current. The electric current and spin polarization is known to be coupled with each other in a 2D electron gas system with Rashba spin splitting. ${ }^{[33]}$ According to ref. [25], a 3D Rashba-like splitted band can be responsible for non-trivial dependence of $g_{\vec{k}}(\vec{B}, \vec{E})$ on the $\vec{B}$ and $\vec{E}$ orientations. Particularly, the calculated Berry curvature showed a fourfold enhancement in magnitude along the $\Gamma-\mathrm{Z}$ direction of the 3D Fermi surface in needle $\mathrm{Cd}_{3} \mathrm{As}_{2}$ crystal. In this case, non-equilibrium electron distribution due to external magnetic field and electric bias can result in very large anomalous velocity contribution to the electron magnetotransport.

It would be helpful to list main features of the $\left(\mathrm{Cd}_{0.6} \mathrm{Zn}_{0.36} \mathrm{Mn}_{0.04}\right)_{3} \mathrm{As}_{2}$ single crystal studied in this work, which make it different from other $\mathrm{Cd}_{3} \mathrm{As}_{2}$ compounds. ${ }^{[1,20,23,34-36]}$ These features should be taken into account to develop the mechanism of the antisymmetric magnetoresistance which is due to a possible contribution of intrinsic anomalous velocity term in the transport equation (expression (4)).

(i) The $\left(\mathrm{Cd}_{1-x} \mathrm{Zn}_{x}\right)_{3} \mathrm{As}_{2}$ system should be considered as solid solutions system consisting of end $\mathrm{Cd}_{3} \mathrm{As}_{2}$ and $\mathrm{Zn}_{3} \mathrm{As}_{2}$ members. It is known that below room temperature $\mathrm{Cd}_{3} \mathrm{As}_{2}$ has a body-center tetragonal structure corresponding to either non-centrosymmetric $\mathrm{I} 4_{1} \mathrm{Cd}$ space group or centrosymmetric I4 1 /acd space group. The $\mathrm{Cd}_{3} \mathrm{As}_{2}$ single crystals having one of these body-center tetragonal structures are usually studied. However, structural transitions from a body-center tetragonal phase to a primitive tetragonal phase with $\mathrm{P}_{2} / \mathrm{nmc}$ symmetry then back to a body-center tetragonal phase take place in the $\left(\mathrm{Cd}_{1-x} \mathrm{Zn}_{x}\right)_{3} \mathrm{As}_{2}$ solid solutions with increasing $x .^{[37]}$ The $\left(\mathrm{Cd}_{0.6} \mathrm{Zn}_{0.36} \mathrm{Mn}_{0.04}\right)_{3} \mathrm{As}_{2}$ single crystal studied in this work did have the primitive tetragonal $\mathrm{P}_{2} / \mathrm{nmc}$ structure.

(ii) The structural $\left(\mathrm{Cd}_{1-x} \mathrm{Zn}_{x}\right)_{3} \mathrm{As}_{2}$ transformations resulting from the $x$ change is accompanied by a chemical-dopingcontrolled transition from a 3D Dirac semimetal to a conventional semiconductor with a critical $x_{\mathrm{C}}=0.38$ point. ${ }^{[37]}$ So, the $\left(\mathrm{Cd}_{0.6} \mathrm{Zn}_{0.36} \mathrm{Mn}_{0.04}\right)_{3} \mathrm{As}_{2}$ composition should be believed to be close enough to $x_{\mathrm{C}}$. It should be also noted that for the semiconductor $\left(\mathrm{Cd}_{1-x} \mathrm{Zn}_{x}\right)_{3} \mathrm{As}_{2}$ compositions, the specific electrical resistivity is already increasing with decreasing temperature. But, the $\left(\mathrm{Cd}_{0.6} \mathrm{Zn}_{0.36} \mathrm{Mn}_{0.04}\right)_{3} \mathrm{As}_{2}$ resistivity is gradually decreasing with decreasing temperature (Figure 3). Hence, the $\left(\mathrm{Cd}_{0.6} \mathrm{Zn}_{0.36} \mathrm{Mn}_{0.04}\right)_{3} \mathrm{As}_{2}$ composition is still below $x_{\mathrm{C}}$. As was mentioned above, a 3D Dirac semimetal with inverted bands is characterized by overlapping two Weyl nodes with opposite chirality in momentum space. In contrast to the Dirac semimetal, the Dirac nodes degeneracy is removed in a Weyl semimetal, that is only individual Weyl bandtouching nodes with opposite topological charges in momentum space will occur. Both the Dirac semimetal and the Weyl semimetal are gapless states. For the $\left(\mathrm{Cd}_{1-x} \mathrm{Zn}_{x}\right)_{3} \mathrm{As}_{2}$ solid solutions, a transition from a gapless Dirac semimetal state into gapped state of conventional semiconductor should be accompanied by forming a gapless Weyl semimetal state. Appearance of a 
linear-in- $B$ contribution into the magnetoresistance is characteristic for the Weyl semimetal.

(iii) The $\mathrm{Mn}$ atoms were additionally used to modify the $\left(\mathrm{Cd}_{1-x} \mathrm{Zn}_{x}\right)_{3} \mathrm{As}_{2}$ properties. Atoms and ions of $\mathrm{Mn}$ are characterizing by a strong spin-orbit interaction, that is also important physical factor resulting in forming a 3D topological Dirac semimetal state. These atoms and ions are often used to prepare so-called diluted magnetic semiconductors possessing with unusual transport properties in applied magnetic field. In this case, by analogy with diluted magnetic semiconductors, $\left(\mathrm{Cd}_{0.6} \mathrm{Zn}_{0.36} \mathrm{Mn}_{0.04}\right)_{3} \mathrm{As}_{2}$ should be reasonably considered as a diluted magnetic Dirac semimetal. It is also important to note that a incorporation of magnetic dopants into topological insulators can break time reversal symmetry. ${ }^{[38]}$

Finally, the unique 3D Rashba-like spin-splitted bands earlier used to explain the antisymmetric magnetoresistance in needle $\mathrm{Cd}_{3} \mathrm{As}_{2}$ crystal were obtained from band calculations with the $\mathrm{Cd}$ antisite defects. ${ }^{[25]}$ These defects are believed to be responsible for breaking both the inversion and rotational symmetry of $\mathrm{Cd}_{3} \mathrm{As}_{2}$. In other words, to get the antisymmetric magnetoresistance, the specific defect structure should be formed. In ref. [25] such kind of defect structure was obviously formed during the material synthesis. Besides the $\mathrm{Cd}$ antisite defects, the defect structure favoring to appearance of both asymmetry and parity violation in the $\left(\mathrm{Cd}_{0.6} \mathrm{Zn}_{0.36} \mathrm{Mn}_{0.04}\right)_{3} \mathrm{As}_{2}$ magnetoresistance can be related to other defects due to the $\mathrm{Zn}$ and $\mathrm{Mn}$ atoms substituted for the $\mathrm{Cd}$ atoms.

One can also conclude that the anomalous velocity term associated with the non-zero Berry curvature is dominant contribution to the antisymmetric magnetoresistance for the 2 orientation, whereas this contribution for the 1 and 3 orientations is small enough and it only results in the asymmetric magnetoresistance (Figure 1).

To account for the magnetoresistance features found in $\left(\mathrm{Cd}_{0.6} \mathrm{Zn}_{0.36} \mathrm{Mn}_{0.04}\right)_{3} \mathrm{As}_{2}$, band calculations taking into account crystal symmetry, exact elemental composition and defect structure should be further done.

\section{Supporting Information}

Supporting Information is available from the Wiley Online Library or from the author.

\section{Acknowledgments}

Vasilii Zakhvalinskii thanks the Ministry of Education and Science of the Russian Foundation for Basic research for the financial support under project No. 17-02-00262A. All of the studies were carried out by the scientific equipment of joint research center "Technologies and Materials" at the Belgorod State University.

\section{Conflict of Interest}

The authors declare no conflict of interest.

\section{Keywords}

Dirac semimetal, magnetoresistance, quantum Hall effect, Shubnikov-de Haas oscillations, Weyl semimetal

Received: August 7, 2018

Revised: September 5, 2018 Published online:

[1] J. S. Blakemore, Solid State Physics. Cambridge University Press, UK 1985, pp. 405-489.

[2] Q. Li, B. T. Liu, Y. F. Hu, J. Chen, H. Gao, L. Shan, H. H. Wen, A. V. Pogrebnyakov, J. M. Redwing, X. X. Xi, Phys. Rev. Lett. 2006, 96, 167003.

[3] M. Schultza, L. Klein, Appl. Phys. Lett. 2007, 91, 151104.

[4] J. Ping, I. Yudhistira, N. Ramakrishnan, S. Cho, S. Adam, M. S. Fuhrer, Phys. Rev. Lett. 2014, 113, 047206.

[5] J. Tian, C. Chang, H. Cao, K. He, X. Ma, Q. Xue, Y. P. Chen, Sci. Rep. 2016, 4, 4859.

[6] Z. Wang, H. Weng, Q. Wu, X. Dai, Z. Fang, Phys. Rev. B 2013, 88, 125427.

[7] Y. Zhao, H. Liu, C. Zhang, H. Wang, J. Wang, Z. Lin, Y. Xing, H. Lu, J. Liu, Y. Wang, S. M. Brombosz, Z. Xiao, S. Jia, X. C. Xie, J. Wang, Phys. Rev. X 2015, 5, 031037.

[8] T. Liang, J. Lin, Q. Gibson, T. Gao, M. Hirschberger, M. Liu, R. J. Cava N. P. Ong, Phys. Rev. Lett. 2017, 118, 136601.

[9] J. Cao, S. Liang, C. Zhang, Y. Liu, J. Huang, Z. Jin, Z.-G. Chen, Z. Wang, Q. Wang, J. Zhao, S. Li, X. Dai, J. Zou, Z. Xia, L. Li, F. Xiu, Nature Commun. 2015, 6, 7779.

[10] Z. J. Xiang, D. Zhao, Z. Jin, C. Shang, L. K. Ma, G. J. Ye, B. Lei, T. Wu, Z. C. Xia, X. H. Chen, Phys. Rev. Lett. 2015, 115, 226401.

[11] R. Sankar, M. Neupane, S.-Y. Xu, C. J. Butler, I. Zeljkovic, I. P. Muthuselvam, F.-T. Huang, S.-T. Guo, S. K. Karna, M.-W. Chu, W. L. Lee, M.-T. Lin, R. Jayavel, V. Madhavan, M. Z. Hasan, F. C. Chou, Sci. Rep. 2015, 5, 12966.

[12] H. Wang, X. Luo, W. Chen, N. Wang, B. Lei, F. Meng, C. Shang, L. Ma, T. Wu, Xi Dai, Z. Wang, X. Chen, Sci. Bull. 2018, 63, 411.

[13] Y. Ando, J. Phys. Soc. Jpn. 2013, 82, 102001.

[14] D. T. Son, B. Z. Spivak, Phys. Rev. B 2013, 88, 104412.

[15] A. A. Zyuzin, A. A. Burkov, Phys. Rev. B 2013, 88, 125110.

[16] S. A. Parameswaran, T. Grover, D. A. Abanin, D. A. Pesin, A. Vishwanath, Phys. Rev. X 2014, 4, 031035.

[17] Y.-Y. Zhang, X.-R. Wang, X. C. Xie, J. Phys.: Condens. Matter 2012, 24, 015004.

[18] D. E. Kharzeev, H.-U. Yee, Phys. Rev. B 2013, 88, 115119.

[19] S. M. Young, S. Zaheer, J. C. Y. Teo, C. L. Kane, E. J. Mele, A. M. Rappe, Phys. Rev. Lett. 2012, 108, 140405.

[20] J. Feng, Y. Pang, D. Wu, Z. Wang, H. Weng, J. Li, X. Dai, Z. Fang, Y. Shi, L. Luy, Phys. Rev. B 2015, 92, 081306.

[21] H. Lu, S. Jia, Front. Phys. 2017, 12, 127211.

[22] J. Zhang, M. Gao, J. Zhang, X. Wang, X. Zhang, M. Zhang, W. Niu, R. Zhang, Y. Xu, Front. Phys. 2018, 13, 137201.

[23] H.-J. Kim, K.-S. Kim, J.-F. Wang, M. Sasaki, N. Satoh, A. Ohnishi, M. Kitaura, M. Yang, L. Li, Phys. Rev. Lett. 2013, 111, 246603.

[24] S. Liang, J. Lin, S. Kushwaha, J. Xing, N. Ni, R. J. Cava, N. P. Ong, Phys. Rev. X 2018, 8, 031002.

[25] S.-T. Guo, R. Sankar, Y.-Y. Chien, T.-R. Chang, H.-T. Jeng, G.-Y. Guo, F. C. Chou, W.-L. Lee, Sci. Rep. 2016, 6, 27487.

[26] D. Schoenberg, Magnetic Oscillations in Metals. Cambridge University Press, London 1984

[27] V. S. Zakhvalinskii, T. B. Nikulicheva, E. Lähderanta, M. A. Shakhov, E. A. Nikitovskaya, S. V. Taran, J. Phys.: Condens. Matter 2017, 29, 455701 . 
[28] H. Cao, J. Tian, I. Miotkowski, T. Shen, J. Hu, Phys. Rev. Lett. 2012, 108,216803

[29] D. Elefant, G. Reiss, C. Baier, Eur. Phys. J. B 1998, 4, 45.

[30] R. Xu, A. Husmann, T. F. Rosenbaum, M. L. Saboungi, J. E. Enderby, P. B. Littlewood, Nature 1997, 390, 57.

[31] A. Husmann, J. B. Betts, G. S. Boebinger, A. Migliori, T. F. Rosenbaum, M. L. Saboungi, Nature 2002, 417, 421.

[32] J. Hu, T. F. Rosenbaum, Nat. Mater. 2008, 7, 697.

[33] R. H. Silsbee, Phys. Rev. B 2001, 63, 155305.

[34] C.-Z. Li, L.-X. Wang, H. Liu, J. Wang, Z.-M. Liao, D.-P. Yu, Nature Commun. 2015, 6, 10137.
[35] J. Cao, S. Liang, C. Zhang, Y. Liu, J. Huang, Z. Jin, Z.-G. Chen, Z. Wang, Q. Wang, J. Zhao, S. Li, X. Dai, J. Zou, Z. Xia, L. Li, F. Xiu, Nature Commun. 2015, 6, 7779.

[36] Z. J. Xiang, D. Zhao, Z. Jin, C. Shang, L. K. Ma, G. J. Ye, B. Lei, T. Wu, Z. C. Xia, X. H. Chen, Phys. Rev. Lett. 2015, 115, 226401.

[37] H. Lu, X. Zhang, Y. Bian, S. Jia, Sci. Rep. 2017, 7, 3148.

[38] S. E. Harrison, L. J. Collins-McIntyre, S. Li, A. A. Baker, L. R. Shelford, Y. Huo, A. Pushp, S. S. P. Parkin, J. S. Harris, E. Arenholz, G. van der Laan, T. Hesjedal, J. Appl. Phys. 2014, 115, 023904. 